\title{
Thirty-four Musa (Musaceae) expressed sequence tag-derived microsatellite markers transferred to Musella lasiocarpa
}

\author{
W.J. Li ${ }^{1,2 *}$ H. Ma ${ }^{1 *}$, Z.H. Li ${ }^{1}$, Y.M. Wan ${ }^{1}$ X.X. Liu ${ }^{1}$ and C.L. Zhou ${ }^{1}$ \\ ${ }^{1}$ Biotechnology Department, Research Institute of Resource Insects, \\ Chinese Academy of Forestry, Kunming, China \\ ${ }^{2}$ Yunnan Reascend Tobacco Technology (Group) Company Limited, Kunming, \\ China \\ *These authors contributed equally to this study. \\ Corresponding author: Z.H. Li \\ E-mail: 1zh4949@163.com
}

Genet. Mol. Res. 11 (3): 2094-2098 (2012)

Received January 9, 2012

Accepted March 30, 2012

Published August 6, 2012

DOI http://dx.doi.org/10.4238/2012.August.6.13

\begin{abstract}
We assembled 31,308 publicly available Musa EST sequences into 21,129 unigenes; 4944 of them contained 5416 SSR motifs. In all, 238 unigenes flanking SSRs were randomly selected for primer design and then tested for amplification in Musella lasiocarpa. Seventy-eight primer pairs were found to be transferable to this species, and 49 displayed polymorphism. A set of 34 polymorphic SSR markers was analyzed in 24 individuals from four wild M. lasiocarpa populations. The mean number of alleles per locus was 3.0, ranging from 2 to 7. The observed and expected heterozygosities per marker ranged from 0.087 to 0.875 (mean 0.503 ) and from 0.294 to 0.788
\end{abstract}


(mean 0.544), respectively. These markers will be of practical use for genetic diversity and quantitative trait loci analysis of M. lasiocarpa.

Key words: EST-SSRs; Molecular markers; Musella; Transferability; Polymorphism

\section{INTRUDUCTION}

Musella lasiocarpa, normally known as Chinese dwarf banana, native to southwestern China, represents the monotypic genus Musella in the family Musaceae (Wu and Kress, 2000). Its showy and lotus-like golden inflorescence with more than eight month anthesis has strongly attracted horticulturists; consequently, it has been introduced into various regions and botanical gardens around the world. Wild populations of M. lasiocarpa are found on cliffs within the watershed of the upper Yangtze River and its tributaries, in northern Yunnan and southern Sichuan Provinces in China. It is now difficult to find wild populations as appropriate habitats have been fragmented due to widespread expansion of agriculture (Ma et al., 2011). However, it is locally common in this region of southwestern China, maintained as a semi-cultivated plant. This taxon is extensively used by farmers as fodder for livestock, for erosion control, and as weaving material (Ma et al., 2011). Plants have been utilized as an edible vegetable, as medicine, in wine making, and even as a resource for honey production (Liu et al., 2003; Long et al., 2008). Considering the high ornamental value and endemism of M. lasiocarpa, we have been committed to investigate the diversity of its wild populations since 2004 and have documented nine wild populations (one of them contains less than 30 tufts) to date. We assessed the conservation status of its wild populations as Endangered, according to IUCN Red List criteria (IUCN, 2001), because the number of population locations and mature plants could fluctuate extremely in the near future.

Microsatellite (SSR) markers have proven to be a powerful tool to investigate genetic diversity, mapping and cultivar identification. However, there have been relatively few reports of SSR markers in M. lasiocarpa (Yang et al., 2009). Expressed sequence tag (EST)-derived SSR markers show a higher level of transferability to closely related species and fewer null alleles than genomic-SSRs and provide an efficient means for SSR screening (La Rota et al., 2005; Cristancho and Escobar, 2008; Li et al., 2010). We found no genetic maps or EST sequences for M. lasiocarpa in GenBank. However, it is reasonable to expect that useful SSR markers could be developed from Musa (Musaceae) EST sequences.

\section{MATERIAL AND METHODS}

Twenty-four individuals from four wild populations of M. lasiocarpa from Yunnan Province, China, were collected to screen for transferability and polymorphism of Musa ESTderived SSR markers. All of them had green petioles, pale green primary veins and yellow bracts. Plant genomic DNA from young leaves was extracted using the CTAB method (Doyle and Doyle, 1990) and stored at $-20^{\circ} \mathrm{C}$.

We obtained 31,308 publicly available Musa EST sequences (11,155 from 
Musa paradisiaca, 5289 from Musa balbisiana, 14,864 from Musa acuminata) from the NCBI database (http://www.ncbi.nlm.nih.gov/nucest) on September 10, 2010 and saved them as Fasta-formatted files. The 31,308 sequences were assembled into 21,129 non-redundant clusters (11,165 for M. acuminata, 5908 for M. paradisiaca and 4056 for M. balbisiana), using the CAP3 software (Huang and Madan, 1999). Unigenes containing SSRs were identified with SSRIT (http://www.gramene.org/db/markers/ssrtool). The minimum length of SSR was defined as di-, tri-, tetra-, penta-, and hexanucleotide (DNRs, TNRs, TeNRs, PNRs, and HNRs) for 6, 4, 3, 3, and 3 subunits, respectively. Across all three species, 5416 SSRs (234 were compound SSRs) were identified in 4944 unigenes. Primer pairs were designed with the help of PRIMER3 (Rozen and Skaletsky, 2000), using the following settings: optimal primer length $20 \mathrm{bp}$ (maximum $25 \mathrm{bp}$, minimum $18 \mathrm{bp}$ ), optimal annealing temperature $(\mathrm{Tm}) 57^{\circ} \mathrm{C}$ (maximum $63^{\circ} \mathrm{C}$, minimum $52^{\circ} \mathrm{C}$, maximum temperature difference $5^{\circ} \mathrm{C}$ ), product size $100-500 \mathrm{bp}$. The CG content varied from 35 to $65 \%$.

The PCR was performed in a $20 \mu \mathrm{L}$ volume containing $25 \mathrm{ng}$ genomic DNA, $0.75 \mathrm{U} \mathrm{Taq}$ DNA polymerase, $1 \mathrm{X}$ PCR buffer (10 mM Tris- $\mathrm{HCl}, \mathrm{pH} 8.0,50 \mathrm{mM} \mathrm{KCl})$, $1.8 \mathrm{mM} \mathrm{MgCl}, 0.13 \mathrm{mM}$ dNTPs (Takara), and $0.25 \mu \mathrm{M}$ of each forward and reserve primers. The PCR was carried out using the following thermal cycling program: initial denaturation at $95^{\circ} \mathrm{C}$ for $4 \mathrm{~min}$, followed by 30 cycles of $95^{\circ} \mathrm{C}$ for $45 \mathrm{~s}, 45 \mathrm{~s}$ at primerspecific annealing temperature of $50^{\circ}$ to $63^{\circ} \mathrm{C}, 72^{\circ} \mathrm{C}$ for $1 \mathrm{~min}$, and a final extension step at $72^{\circ} \mathrm{C}$ for $10 \mathrm{~min}$. PCR products were separated on $8 \%$ non-denatured polyacrylamide gel and then stained with ethidium bromide. The successful PCR products were subsequently sized on an ABI PRISM 3730xl Genetic Analyzer (Applied Biosystems, Foster City, CA, USA). The forward primers were labeled at the 5'-end with an FAM fluorescent dye.

The number of alleles $\left(N_{\mathrm{A}}\right)$, linkage disequilibrium, observed $\left(H_{\mathrm{O}}\right)$ and expected $\left(H_{\mathrm{E}}\right)$ heterozygosities were calculated by the PopGen version 1.31 software (http://www. ualberta.ca/ fyeh/). The fitness of genotypic frequency to the Hardy-Weinberg equilibrium (HWE) was determined using FSTAT version 2.9.3 (Goudet, 1995).

\section{RESULTS AND DISCUSSION}

In all, 78 of the 238 Musa EST-derived SSR primers successfully amplified PCR products at close to the size expected in M. lasiocarpa. The remaining primer pairs amplified no products, or gave weak or indistinct bands, or gave obviously larger sizes than expected. In the 34 polymorphic EST-SSR primer pairs that were identified, the $N_{\mathrm{A}}$ ranged from 2 to 7, with a mean of 3.0. Compared with the loci developed from the genome (Yang et al., 2009), the $N_{\mathrm{A}}$ per locus was relatively low. The $H_{\mathrm{O}}$ ranged from 0.087 to 0.875 (mean 0.503), and $H_{\mathrm{E}}$ ranged from 0.294 to 0.788 (mean 0.544) (Table 1). Among the 34 microsatellite loci, nine showed significant deviation from HWE $(\mathrm{P}<0.01)$, indicating the possibility of null alleles or sampling problems. All loci were in linkage disequilibrium. The addition of 34 polymorphic microsatellite markers to the genomic-SSR markers (Yang et al., 2009) will prove valuable for future analysis of genetic diversity of wild populations and study of quantitative traits of $M$. lasiocarpa. 
Table 1. Transferability of 34 Musa EST-SSR markers to Musella lasiocarpa.

\begin{tabular}{|c|c|c|c|c|c|c|c|c|c|}
\hline \multirow[t]{2}{*}{ Locus } & \multirow{2}{*}{$\begin{array}{l}\text { Accession } \\
\text { No. }\end{array}$} & \multirow{2}{*}{$\begin{array}{l}\text { Repeat } \\
\text { motif }\end{array}$} & \multirow[t]{2}{*}{ Primer sequences $\left(5^{\prime}-3^{\prime}\right)$} & \multirow[t]{2}{*}{$\mathrm{Ta}\left({ }^{\circ} \mathrm{C}\right)$} & \multicolumn{5}{|c|}{ M. lasiocarpa } \\
\hline & & & & & Size range & $N_{\mathrm{A}}$ & $H_{\mathrm{O}}$ & $H_{\mathrm{E}}$ & $\mathrm{P}_{\text {HWE }}$ \\
\hline$\overline{\text { Smu2 }}$ & FL641808 & $(\mathrm{CTC})_{5}$ & $\begin{array}{l}\text { F: TTCCAACCTGCCACCCTCCT } \\
\text { R: GCCTTTGATTCCGGCGAAGC }\end{array}$ & 56 & $255-273$ & 2 & 0.708 & 0.467 & 0.991 \\
\hline Smu10 & FL646854 & $(\mathrm{CTT})_{5}$ & $\begin{array}{l}\text { F: TGCGGAAGTGCAGTGTGACC } \\
\text { R: TGCACTTGCGAGCAGACGTT }\end{array}$ & 54 & $199-220$ & 5 & 0.542 & 0.788 & $0.001 *$ \\
\hline Smu19 & FL648830 & $(\mathrm{GAGTCT})_{6}$ & $\begin{array}{l}\text { F: TCATGCGCTCAAGCTCCGTT } \\
\text { R: AAGGGTCCGATTCTTCGGCG }\end{array}$ & 54 & $226-250$ & 4 & 0.167 & 0.694 & $0.000^{*}$ \\
\hline Smu24 & FL650445 & $(\mathrm{GAA})_{7}$ & $\begin{array}{l}\text { F: GGGATAGAGCAGCAGACCGC } \\
\text { R: CTGGCAGCGAACCGATCCAT }\end{array}$ & 61 & $164-179$ & 4 & 0.583 & 0.715 & 0.048 \\
\hline Smu29 & FL648383 & $(\mathrm{ATCG})_{4}$ & $\begin{array}{l}\text { F: GCTTCATGGAGCTGCGGGAG } \\
\text { R: ACGATGAAGGAGGCCGAGGA }\end{array}$ & 51 & $288-312$ & 2 & 0.458 & 0.361 & 0.771 \\
\hline Smu148 & FL649365 & $(\mathrm{GAT})_{6}$ & $\begin{array}{l}\text { F: GCAGAAACAGGCACCGGGTA } \\
\text { R: TGTGTTCGGGTGCTCCATGT }\end{array}$ & 57 & $219-228$ & 2 & 0.750 & 0.507 & 0.980 \\
\hline Smu153 & FL646992 & $(\mathrm{GA})_{13}$ & $\begin{array}{l}\text { F: GAGTCAGTGAGCGAGGAGCG } \\
\text { R: AGGGCGAGGGTTCTTCTCGT }\end{array}$ & 53 & $211-215$ & 3 & 0.208 & 0.543 & $0.000 *$ \\
\hline Smu155 & FL650274 & $(\mathrm{AT})_{9}$ & $\begin{array}{l}\text { F: GAGATGGCCTGTGGCGTCAA } \\
\text { R: GAAAGCTTCTCGAGGGCCGA }\end{array}$ & 55 & $175-179$ & 2 & 0.348 & 0.510 & 0.024 \\
\hline Smu157 & FL649749 & $(\mathrm{GTG})_{4}$ & $\begin{array}{l}\text { F: GAGACCCGACACTTCGGTGG } \\
\text { R: TCCGTGTGCCGAAAGGTCTG }\end{array}$ & 53 & $202-214$ & 3 & 0.087 & 0.552 & $0.000^{*}$ \\
\hline Smu158 & FL649571 & $(\mathrm{GTG})_{4}$ & $\begin{array}{l}\text { F: GACGGAGACCCGACACTTCG } \\
\text { R: AAGCCCGAGATCTTGCCTGC }\end{array}$ & 52 & 293-296 & 2 & 0.833 & 0.497 & 0.999 \\
\hline Smu162 & FL651169 & $(\mathrm{AG})_{20}$ & $\begin{array}{l}\text { F: GAAGCGGCGACTTTGATCGC } \\
\text { R: AGTCGGGCTTCTTGTGAGCG }\end{array}$ & 54 & $195-215$ & 7 & 0.417 & 0.616 & $0.005^{*}$ \\
\hline Smu180 & FL646390 & $(\mathrm{TCG})_{5}$ & $\begin{array}{l}\text { F: CGCTCCACTCCACGCTTCTT } \\
\text { R: ACGATATGCGGTGCCCATCC }\end{array}$ & 52 & $211-236$ & 4 & 0.583 & 0.481 & 0.871 \\
\hline Smu181 & FL647640 & $(\mathrm{TCG})_{5}$ & $\begin{array}{l}\text { F: CGCTCCACTCCACGCTTCTT } \\
\text { R: GGTGCCCATCCTTCTCCGTG }\end{array}$ & 63 & $212-230$ & 3 & 0.636 & 0.661 & 0.384 \\
\hline Smu182 & FL646375 & $(\mathrm{GCC})_{8}$ & $\begin{array}{l}\text { F: CGCCTCCGAAACCCTTCCTC } \\
\text { R: CCGCGTTCCCACAGTATCCG }\end{array}$ & 54 & $189-192$ & 2 & 0.391 & 0.476 & 0.303 \\
\hline Smu184 & FL651442 & $(\mathrm{CCT})_{8}$ & $\begin{array}{l}\text { F: CGAGTCGACGCCTGTGAGAG } \\
\text { R: ACTGCTTGATGGCGTCCTGG }\end{array}$ & 51 & $147-171$ & 4 & 0.667 & 0.687 & 0.324 \\
\hline Smu193 & FL647754 & $(\mathrm{CCT})_{9}$ & $\begin{array}{l}\text { F: CCGTCTCTTCCGCTGCTCTC } \\
\text { R: CTGAGCCCGTCGAATCTCGG }\end{array}$ & 55 & $178-187$ & 2 & 0.522 & 0.394 & 0.863 \\
\hline Smu198 & FL650994 & $(\text { TAAACC })_{8}$ & $\begin{array}{l}\text { F: CATCCTCGTGGTGCAGGCAA } \\
\text { R: CCACCAACAACGAGGTAGCCA }\end{array}$ & 56 & $229-241$ & 2 & 0.542 & 0.503 & 0.690 \\
\hline Smu221 & FL650890 & $(\mathrm{TCGA})_{4}$ & $\begin{array}{l}\text { F: CAGCGAGTCAGTGAGCGAGG } \\
\text { R: CTCTGCGATCTGGTCGTCGG }\end{array}$ & 54 & 194-202 & 3 & 0.261 & 0.564 & $0.001 *$ \\
\hline Smu237 & FL646654 & $(\mathrm{CTG})_{7}$ & $\begin{array}{l}\text { F: ACCACTGGCTGGGTAGGCAT } \\
\text { R: TGCATTCTCAACAAGAAATGAAGGT }\end{array}$ & 50 & $201-225$ & 5 & 0.583 & 0.752 & $0.003^{*}$ \\
\hline Smu239 & FL646915 & $(\mathrm{AGA})_{12}$ & $\begin{array}{l}\text { F: ACAAGATGAGGCCACTGCGG } \\
\text { R: ACAAACGCGAACCCTCTGCT }\end{array}$ & 51 & $295-307$ & 2 & 0.667 & 0.454 & 0.980 \\
\hline Mms10 & FL646660 & $(\mathrm{TCA})_{8}(\mathrm{GT})_{8}$ & $\begin{array}{l}\text { F: GCTTTCGGCCTCGGAGAAGT } \\
\text { R: GAGAGAGAGAGAGAACACAGTTCA }\end{array}$ & 53 & $135-147$ & 4 & 0.652 & 0.737 & 0.124 \\
\hline Musf8 & DN238224 & $(\mathrm{TC})_{14}$ & $\begin{array}{l}\text { F: CACTGCACCTCGGTGGCAAA } \\
\text { R: TCGATCCACCTGAGCAACACGA }\end{array}$ & 51 & $214-218$ & 3 & 0.565 & 0.670 & 0.102 \\
\hline Musf21 & FL665190 & $(\mathrm{TTC})_{11}$ & $\begin{array}{l}\text { F: TCCACCAATAGCAGGCAACACC } \\
\text { R: CCATGCCCAAGCTTCCGCTAA }\end{array}$ & 52 & $233-242$ & 3 & 0.174 & 0.618 & $0.000^{*}$ \\
\hline Musf22 & FL665824 & $(\mathrm{TG})_{19}$ & $\begin{array}{l}\text { F: CCGTCACCCATCCCTTGGGTA } \\
\text { R: ACCGCGTTCATCACCCGTTT }\end{array}$ & 54 & $187-191$ & 2 & 0.667 & 0.507 & 0.905 \\
\hline Musf24 & FL663908 & $(\mathrm{AGA})_{11}$ & $\begin{array}{l}\text { F: GCAGCAGAGTGATCAAAGGAGGC } \\
\text { R: CGCATGGAACCACCGGACAA }\end{array}$ & 59 & $233-239$ & 2 & 0.261 & 0.294 & 0.217 \\
\hline Musf25 & FL663530 & $\left(\right.$ AGATGA) ${ }_{6}$ & $\begin{array}{l}\text { F: TGGAGGGACTTGCAGCCCAA } \\
\text { R: GCCACGAACGCACAACCTCA }\end{array}$ & 55 & $157-175$ & 3 & 0.783 & 0.618 & 0.949 \\
\hline Musf29 & ES433855 & $(\mathrm{CTC})_{10}$ & $\begin{array}{l}\text { F: TGTAAGCGCAACCCTGCCAC } \\
\text { R: TCAGCTGATTTGACGCGGGC }\end{array}$ & 54 & $214-226$ & 3 & 0.875 & 0.547 & 1.000 \\
\hline Musf30 & FL660562 & $(\mathrm{AG})_{12}$ & $\begin{array}{l}\text { F: CGGTGTCAGTAGCAGCAGTGG } \\
\text { R: CGCTTACATCGGCGAGCGAA }\end{array}$ & 60 & $169-173$ & 2 & 0.583 & 0.479 & 0.776 \\
\hline Musf31 & FL660501 & $(\mathrm{GAA})_{8}$ & $\begin{array}{l}\text { F: ATCAGCGAGTCGTCGGAGCA } \\
\text { R: GCAGCAGCAGCAGCTAACGA }\end{array}$ & 58 & 186-192 & 2 & 0.250 & 0.422 & $0.009^{*}$ \\
\hline Musf34 & ES433050 & $(\mathrm{GAA})_{8}$ & $\begin{array}{l}\text { F: TGGCGATGCCCAGGAACTCA } \\
\text { R: TCGAGACGTCCGTCGACAAGT }\end{array}$ & 52 & $149-152$ & 2 & 0.208 & 0.311 & 0.022 \\
\hline
\end{tabular}

Continued on next page 
Table 1. Continued.

\begin{tabular}{|c|c|c|c|c|c|c|c|c|c|}
\hline \multirow[t]{2}{*}{ Locus } & \multirow{2}{*}{$\begin{array}{l}\text { Accession } \\
\text { No. }\end{array}$} & \multirow{2}{*}{$\begin{array}{l}\text { Repeat } \\
\text { motif }\end{array}$} & \multirow[t]{2}{*}{ Primer sequences $\left(5^{\prime}-3^{\prime}\right)$} & \multirow[t]{2}{*}{$\mathrm{Ta}\left({ }^{\circ} \mathrm{C}\right)$} & \multicolumn{5}{|c|}{ M. lasiocarpa } \\
\hline & & & & & Size range & $N_{\mathrm{A}}$ & $H_{\mathrm{O}}$ & $H_{\mathrm{E}}$ & $\mathrm{P}_{\mathrm{HWE}}$ \\
\hline Musf37 & ES436430 & $(\mathrm{CAG})_{8}$ & $\begin{array}{l}\text { F: CAGCTTCAGCCACAGTCGCA } \\
\text { R: GCATGTTGGTTGGCCCTGCT }\end{array}$ & 62 & $238-247$ & 2 & 0.609 & 0.510 & 0.890 \\
\hline Musf39 & ES432796 & $(\mathrm{AG})_{14}$ & $\begin{array}{l}\text { F: CGGTGCCAATGGTGCAAACG } \\
\text { R: AGCATGGCTGAGGTAGTCGCA }\end{array}$ & 60 & $182-192$ & 4 & 0.591 & 0.606 & 0.364 \\
\hline Musf41 & ES432772 & $(\mathrm{AG})_{16}$ & $\begin{array}{l}\text { F: CCGATCTCCCATGGCAGCAAA } \\
\text { R: TGCATCCGCGACGATGTCTG }\end{array}$ & 56 & $248-272$ & 4 & 0.667 & 0.646 & 0.491 \\
\hline Musf50 & DN239657 & $(\mathrm{GCC})_{10}$ & $\begin{array}{l}\text { F: GCTCGCCAACGGTAATGGCA } \\
\text { R: AAGGTGAGCCAGAGGCAGCA }\end{array}$ & 56 & $177-186$ & 2 & 0.273 & 0.304 & 0.363 \\
\hline
\end{tabular}

${ }^{*}$ Significant deviation from Hardy-Weinberg equilibrium $(\mathrm{P}<0.01) . \mathrm{Ta}=$ annealing temperature; $N_{\mathrm{A}}=$ number of alleles; $H_{\mathrm{O}}=$ observed heterozygosity; $H_{\mathrm{E}}=$ expected heterozygosity.

\section{ACKNOWLEDGMENTS}

Research supported by the Special Fund for Forestry Research in the Public Interest Granted by the State Forestry Administration of China (\#200904053) and the Yunnan Natural Science Foundation (\#2009ZC188M).

\section{REFERENCES}

Cristancho M and Escobar C (2008). Transferability of SSR markers from related Uredinales species to the coffee rust Hemileia vastatrix. Genet. Mol. Res. 7: 1186-1192.

Doyle JJ and Doyle JL (1990). Isolation of plant DNA from fresh tissue. Focus 12: 13-15.

Goudet J (1995). FSTAT (Version 1.2): A computer program to calculate F-statistics. J. Hered. 86: 485-486.

Huang X and Madan A (1999). CAP3: A DNA sequence assembly program. Genome Res. 9: 868-877.

IUCN (2001). IUCN Red List Categories and Criteria, Version3.1. Prepared by the IUCN Species Survival Commission. IUCN, Gland, Switzerland, and Cambridge.

La Rota M, Kantety RV, Yu JK and Sorrells ME (2005). Nonrandom distribution and frequencies of genomic and ESTderived microsatellite markers in rice, wheat, and barley. BMC Genomics 6: 23.

Li XY, Shangguan LF, Song CN, Wang C, et al. (2010). Analysis of expressed sequence tags from Prunus mume flower and development of simple sequence repeat markers. BMC Genet. 11: 66.

Liu AZ, Kress WJ and Long CL (2003). Customary use and conservational attention to Musella lasiocarpa (Musaceae): a monotypic genus endemic to China. Econ. Bot. 57: 279-281.

Long CL, Selena A, Wang XR, Liu YT, et al. (2008). Why Musella lasiocarpa (Musaceae) is used in Southwest China to feed pigs. Econ. Bot. 62: 182-186.

Ma H, Pan QJ, Wang L, Li ZH, et al. (2011). Musella lasiocarpa var. rubribracteata (Musaceae), a new variety from Sichuan, China. Novon 21: 349-353.

Rozen S and Skaletsky HJ (2000). Primer 3: Bioinformatics Methods and Protocols. In: Methods in Molecular Biology (Krawetz S and Misener S, eds.). Humana Press, New Jersey, 365-386. Available at [http://frodo.wi.mit.edu/ primer3/]. Accessed November 1, 2010.

Wu DL and Kress WJ (2000). Flora of China: Musaceae. In: Flagellariaceae through Marantaceae (Wu CY and Raven PH, eds.). Vol. 24. Science Press, Beijing, 314-318.

Yang CY, Huang Y and Long CL (2009). Isolation and characterization of 17 polymorphic microsatellite loci for Musella lasiocarpa (Musaceae). HortScience 44: 2041-2042. 